\title{
Diagnósticos de enfermagem em pacientes neurológicos: estudo documental
}

\author{
Nursing diagnoses in neurological patients: \\ a documentary study
}

\author{
Francisco Mayron Morais Soares ${ }^{1}$ (1) \\ Kirley Kethellen Batista Mesquita ${ }^{2}$ (1) \\ Lana Eduarda Silva Praciano Teles ${ }^{3}$ (1)
}

\section{Carlos Lucas Damasceno Pequeno 4 (1) Davi Santos Magalhães ${ }^{5}$ (1) Julyana Gomes Freitas ${ }^{6}$ (D)}

\author{
'Autor para correspondência. Faculdade UNINTA Itapipoca (Itapipoca). Ceará, Brasil. mayronenfo@gmail.com \\ Universidade Federal do Ceará. (Fortaleza). Ceará, Brasil. kirleybatista@gmail.com \\ 3,5Faculdade UNINTA Itapipoca (Itapipoca). Ceará, Brasil. lanaeduarda2011@hotmail.com, davisantos1121246@gamil.com \\ 4,6Universidade de Fortaleza (Fortaleza) Ceará, Brasil. carloslucasdamasceno@gmail.com, julyana.gomes@unifor.br
}

RESUMO | OBJETIVO: Identificar o perfil sociodemográfico e clínico e os diagnósticos de enfermagem em pacientes neurológicos. MÉTODO: Estudo documental realizado entre dezembro de 2017 e março de 2018, com 184 prontuários em um hospital terciário da região nordeste do Brasil. Os dados foram coletados mediante instrumento com perfil sociodemográfico e clínico e apresentados por meio da estatística descritiva. RESULTADOS: A maioria dos pacientes era do sexo masculino e com diagnóstico médico de traumatismo cranioencefálico. Foi observado um total de 25 títulos de diagnósticos de enfermagem, e os que mais prevaleceram foram glicemia instável, confusão aguda, queda, déficit do autocuidado, deambulação prejudicada e conforto prejudicado. Percebe-se a predominância dos diagnósticos de enfermagem para os pacientes neurológicos nos domínios de nutrição e autocuidado. CONCLUSÃO: Os diagnósticos de enfermagem identificados neste estudo podem contribuir para o incremento de indicadores sensíveis à prática de enfermagem e favorece a assistência direcionada as necessidades do paciente.

DESCRITORES: Autocuidado. Diagnóstico de Enfermagem. Transtorno neurológico.

\begin{abstract}
OBJECTIVE: To identify the sociodemographic and clinical profile and nursing diagnoses in neurological patients. METHOD: Documentary study carried out between December 2017 and March 2018, with 184 medical records in a tertiary hospital in the northeast region of Brazil. Data were collected using an instrument with a sociodemographic and clinical profile and presented through descriptive statistics. RESULTS: Most patients were male and medically diagnosed with traumatic brain injury. A total of 25 nursing diagnosis titles were observed, and the most prevalent were unstable blood glucose, acute confusion, falls, self-care deficit, impaired walking and impaired comfort. It is noticed the predominance of nursing diagnoses for neurological patients in the domains of nutrition and self-care. CONCLUSION: The nursing diagnoses identified in this study can contribute to the increase of sensitive indicators for nursing practice and favor assistance directed to the patient's needs.
\end{abstract}

DESCRIPTORS: Self-care. Nursing Diagnosis. Neurological disorder. 


\section{Introdução}

As doenças neurológicas são definidas por complicações que afetam o sistema nervoso, ou seja, cérebro, medula espinhal e/ou nervos periféricos, resultantes de traumas diversos e infecções. Têm-se como principais afecções neurológicas o traumatismo cranioencefálico, o acidente vascular cerebral e o traumatismo raquimedular. ${ }^{1}$

As doenças cerebrovasculares são consideradas um problema de saúde pública, sendo as condições socioeconômicas determinantes para o aumento, sobretudo nos países em desenvolvimento., No Brasil, dentre as doenças cardiovasculares, a cerebrovascular é uma das doenças mais negligenciadas do país, em que pode acarretar incapacidade física e apresentar elevada taxa de incidência e mortalidade. 3 Conforme a literatura, os traumas são uma das principais causas de morte ou incapacidade funcional na projeção para os próximos 10 anos. ${ }^{4}$

A abordagem aos pacientes neurológicos precisa ser holística, integral e humanizada, com vários fatores contribuintes relacionados à qualidade de vida. $\mathrm{O}$ profissional de enfermagem, com o paciente, deve identificar déficits de capacidade no atendimento das necessidades individuais de autocuidado, procurando desenvolver nesses indivíduos os potenciais já existentes para essa prática. .5

Diante do exposto, é dever dos profissionais de saúde e, insere-se o enfermeiro, identificar e tratar os fenômenos decorrentes das afecções neurológicas com implantação de atitudes e competências estratégicas que coincidam com a assistência de enfermagem, de forma holística e humanizada, onde as necessidades dos pacientes sejam atendidas. ${ }^{6}$
O processo de Enfermagem (PE) é um modelo assistencial que objetiva contribuir para a execução profissional de enfermagem como ciência. O PE pode ser composto por cinco etapas: coleta dos dados, diagnóstico de enfermagem, planejamento do cuidado, implementação e avaliação. Desse modo, favorece ações interdependentes e interdisciplinares, melhora a comunicação da equipe de enfermagem e permite que o enfermeiro possa continuar o raciocínio clínico já iniciado pela equipe. Sua utilização favorece a otimização dos cuidados prestados e promove uma maior segurança do paciente. ${ }^{--9}$

A aplicabilidade da Sistematização da Assistência de Enfermagem (SAE) e a implementação do Processo de Enfermagem (PE) são consonantes com um acompanhamento holístico do paciente, onde todas as suas necessidades são atendidas. No Brasil, tais ações são regulamentadas pela Resolução do COFEN $n^{\circ} 358 / 2009.10$

Em busca na literatura, como diagnóstico situacional, percebeu-se que há escassez de estudos acerca dos diagnósticos de enfermagem em pacientes neurológicos. Devido à escassez de estudos no panorama (inter)nacional, esta pesquisa se torna relevante, visto que fornece subsídios para os profissionais de enfermagem acerca dos diagnósticos de enfermagem mais presentes em pacientes neurológicos e permite a avaliação acurada do paciente e, assim, um maior entendimento do seu estado de saúde; além de contribuir na prática clínica baseada em evidências.

Ante ao exposto, o objetivo deste estudo foi identificar o perfil sociodemográfico e clínico e os diagnósticos de enfermagem em pacientes neurológicos. 


\section{Método}

Estudo documental, retrospectivo, com abordagem quantitativa, desenvolvido entre dezembro de 2017 e março de 2018 em unidades de neurologia de hospital terciário. O hospital em questão contempla duas unidades de internações exclusivas aos pacientes neurológicos. Este é considerado o maior centro médico de urgência e emergência, além de ser referência especializada para atendimento aos pacientes (vítimas) de trauma no próprio estado. ${ }^{10}$

A população do estudo foi composta por 355 prontuários de pacientes internados no ano de 2017 em unidade de neurologia. Destes, 184 prontuários foram selecionados de forma probabilística pelo cálculo de população finita. Para compor a amostra foi necessário: ter diagnóstico de alterações neurológicas conforme a Classificação Internacional de Doenças (CID-10) presente no prontuário; ter, no mínimo, 12 horas de internamento nas respectivas unidades. Como critérios de exclusão, os prontuários incompletos e/ou ilegíveis foram retirados da amostra.

A coleta de dados foi realizada por enfermeiros e bolsistas de iniciação científica. Ocorreu por instrumento construído pelos próprios autores, com variáveis referentes ao perfil sociodemográfico: (sexo, idade, cidade de origem, procedência e religião), e perfil clínico: (comorbidades, motivo da internação, suspeita diagnóstica, estado geral de saúde na admissão, dias de internação e desfecho). O preenchimento do formulário realizou-se através do levantamento das informações contidas nos prontuários utilizados pela própria instituição.

Para tanto, a coleta de dados foi realizada com diagnósticos de enfermagem elencados pelos enfermeiros das unidades de neurologia coletados em documento de registo do próprio hospital. Ressalta-se que esse instrumento foi previamente validado por uma aplicação piloto com 10 prontuários, os quais não fizeram parte da amostra.
Os diagnósticos encontrados nos pacientes internados em unidades de neurologia serão apresentados nos resultados conforme a NANDA-I.

Atendeu-se aos princípios éticos que regem as pesquisas com seres humanos, conforme preconiza a Resolução n ${ }^{\circ} 466 / 12$ do Conselho Nacional de Saúde, aprovou-se este estudo pelo Comitê de Ética em Pesquisa Institucional.

\section{Resultados}

Dentre os pacientes, a maioria era do sexo masculino $(83,2 \%)$, a faixa etária de maior prevalência foi de adultos menores de 40 anos $(58,2 \%)$, de etnia parda $(77,7 \%)$, advindos do interior do estado $(60,3 \%)$ e como principal desfecho, alta hospitalar (88\%).

Em relação ao diagnóstico médico conforme a Classificação Estatística Internacional de Doenças e Problemas Relacionados à Saúde (CID-10), o traumatismo cranioencefálico (TCE) ou traumatismo cerebral difuso (S06.2), predominou-se em 54,8\% dos pacientes internados no período de estudo, e tempo de permanência superior a 20 dias $(51,1 \%)$.

Quanto ao perfil clínico em relação aos sistemas morfofuncionais, 54,9\% dos pacientes apresentaram escala de coma de Glasgow leve, na avaliação pupilar houve predominância de pupilas isocóricas $(43,5 \%)$. Em relação ao sistema cardiovascular, 59,2\% dos pacientes encontravam-se normorcardicos, e $48,9 \%$ normotenso. No sistema respiratório, $65,4 \%$ dos pacientes permaneceram eupneicos. Em relação ao sistema gastrointestinal, $23,9 \%$ dos pacientes utilizaram algum dispositivo, sendo que o principal foi a sonda nasogástrica, tanto para alimentação como para medicações de uso habitual. Com relação ao sistema urinário, houve predominância do cateter vesical de demora $(51,1 \%)$. 
Quadro 1. Distribuição dos títulos de diagnósticos de enfermagem identificados em paciente neurológicos internados e Fatores Relacionados conforme os domínios da NANDA-I. 2019 (continua)

\begin{tabular}{|c|c|c|c|c|}
\hline Diagnósticos de Enfermagem & $\mathbf{n}$ & $\%$ & Fator Relacionado & Características definidoras \\
\hline \multicolumn{5}{|l|}{ Domínio 2: } \\
\hline \multicolumn{5}{|l|}{ Classe 1} \\
\hline $\begin{array}{l}\text { Nutrição desequilibrada menor } \\
\text { que as necessidades corporais }\end{array}$ & 88 & 47,8 & $\begin{array}{l}\text { Incapacidade para ingerir } \\
\text { alimentos }\end{array}$ & $\begin{array}{l}\text { Ingestão de alimentos menor } \\
\text { que a ingestão diária } \\
\text { recomendada }\end{array}$ \\
\hline Deglutição prejudicada & 44 & 23,9 & $\begin{array}{l}\text { História de alimentação por } \\
\text { sonda }\end{array}$ & $\begin{array}{l}\text { Ação ineficaz da língua na } \\
\text { formação do bolo alimentar }\end{array}$ \\
\hline \multicolumn{5}{|l|}{ Classe 4} \\
\hline Risco de glicemia instável & 184 & 100 & Ingestão alimentar insuficiente & \\
\hline \multicolumn{5}{|l|}{ Classe 5} \\
\hline Risco de desequilíbrio eletrolítico & 184 & 100 & Diarreia & \\
\hline Volume de líquidos excessivos & 52 & 28,3 & $\begin{array}{l}\text { Mecanismo regulador } \\
\text { comprometido }\end{array}$ & Desequilíbrio eletrolítico \\
\hline \multicolumn{5}{|l|}{ Domínio 3} \\
\hline \multicolumn{5}{|l|}{ Classe 1} \\
\hline Eliminação urinária prejudicada & 98 & 53,3 & Múltiplas causas & $\begin{array}{l}\text { Oligúria conforme balanço } \\
\text { hídrico }\end{array}$ \\
\hline \multicolumn{5}{|l|}{ Classe 2} \\
\hline Risco de constipação & 184 & 100 & $\begin{array}{l}\text { Ingestão insuficiente de fibras / } \\
\text { Motilidade gastrintestinal } \\
\text { diminuída / Mudanças nos } \\
\text { hábitos alimentares }\end{array}$ & \\
\hline \multicolumn{5}{|l|}{ Domínio 4} \\
\hline \multicolumn{5}{|l|}{ Classe 2} \\
\hline Deambulação prejudicada & 184 & 100 & $\begin{array}{l}\text { Equilíbrio prejudicado / Alteração } \\
\text { da função cognitiva / Prejuízo } \\
\text { neuromuscular }\end{array}$ & $\begin{array}{l}\text { Capacidade prejudicada de } \\
\text { andar uma distância } \\
\text { necessária }\end{array}$ \\
\hline Mobilidade física prejudicada & 184 & 100 & $\begin{array}{l}\text { Alteração da função cognitiva / } \\
\text { Prejuízo neuromuscular / Dor }\end{array}$ & Desconforto \\
\hline \multicolumn{5}{|l|}{ Classe 4} \\
\hline $\begin{array}{l}\text { Risco de perfusão tissular } \\
\text { cerebral ineficaz }\end{array}$ & 184 & 100 & Lesão cerebral & \\
\hline $\begin{array}{l}\text { Déficit do autocuidado para } \\
\text { alimentação }\end{array}$ & 184 & 100 & $\begin{array}{l}\text { Alteração da função cognitiva / } \\
\text { Desordens perceptivas / Prejuízo } \\
\text { neuromuscular }\end{array}$ & $\begin{array}{l}\text { Capacidade prejudicada de } \\
\text { engolir o alimento }\end{array}$ \\
\hline $\begin{array}{l}\text { Déficit do autocuidado para } \\
\text { banho }\end{array}$ & 184 & 100 & $\begin{array}{l}\text { Alteração da função cognitiva / } \\
\text { Desordens perceptivas / Prejuízo } \\
\text { neuromuscular }\end{array}$ & $\begin{array}{l}\text { Capacidade prejudicada de } \\
\text { acessar o banheiro }\end{array}$ \\
\hline $\begin{array}{l}\text { Déficit do autocuidado para } \\
\text { higiene íntima }\end{array}$ & 184 & 100 & $\begin{array}{l}\text { Alteração da função cognitiva / } \\
\text { Desordens perceptivas / Prejuízo } \\
\text { neuromuscular }\end{array}$ & $\begin{array}{l}\text { Capacidade prejudicada de } \\
\text { chegar ao vaso sanitário }\end{array}$ \\
\hline
\end{tabular}


Quadro 1. Distribuição dos títulos de diagnósticos de enfermagem identificados em paciente neurológicos internados e Fatores Relacionados conforme os domínios da NANDA-I. 2019 (conclusão)

\begin{tabular}{|c|c|c|c|c|}
\hline Diagnósticos de Enfermagem & $\mathbf{n}$ & $\%$ & Fator Relacionado & Características definidoras \\
\hline $\begin{array}{l}\text { Déficit do autocuidado para } \\
\text { vestir-se }\end{array}$ & 184 & 100 & $\begin{array}{l}\text { Alteração da função cognitiva / } \\
\text { Desordens perceptivas / Prejuízo } \\
\text { neuromuscular }\end{array}$ & $\begin{array}{l}\text { Capacidade prejudicada de } \\
\text { vestir cada um dos itens do } \\
\text { vestuário }\end{array}$ \\
\hline \multicolumn{5}{|l|}{ Domínio 5} \\
\hline \multicolumn{5}{|l|}{ Classe 4} \\
\hline Confusão aguda & 53 & 28,8 & Delirium & Percepções incorretas \\
\hline \multicolumn{5}{|l|}{ Domínio 9} \\
\hline \multicolumn{5}{|l|}{ Classe 1} \\
\hline $\begin{array}{l}\text { Síndrome do estresse por } \\
\text { mudança }\end{array}$ & 2 & 1 & $\begin{array}{l}\text { Estado de saúde comprometido / } \\
\text { Funcionamento psicossocial } \\
\text { prejudicada }\end{array}$ & $\begin{array}{l}\text { Preocupação quanto a } \\
\text { mudança }\end{array}$ \\
\hline Risco do estresse por mudança & 184 & 100 & $\begin{array}{l}\text { Aconselhamento insuficiente / } \\
\text { Mudança ambiental significativa }\end{array}$ & \\
\hline Síndrome do trauma de estupro & 1 & 0,5 & Estupro & $\begin{array}{l}\text { Constrangimento / Alteração } \\
\text { no padrão de sono }\end{array}$ \\
\hline \multicolumn{5}{|l|}{ Domínio 11} \\
\hline \multicolumn{5}{|l|}{ Classe 1} \\
\hline Risco de infecção & 184 & 100 & Procedimentos invasivos & \\
\hline \multicolumn{5}{|l|}{ Classe 2} \\
\hline Risco de queda & 184 & 100 & $\begin{array}{l}\text { Alteração da função cognitiva / } \\
\text { Mobilidade prejudicada }\end{array}$ & \\
\hline Risco de úlcera por pressão & 184 & 100 & $\begin{array}{l}\text { Escore da Escala de Braden / } \\
\text { Mobilização física / Redução da } \\
\text { perfusão tissular / Umidade da } \\
\text { pele }\end{array}$ & \\
\hline $\begin{array}{l}\text { Risco de integridade da pele } \\
\text { prejudicada }\end{array}$ & 184 & 100 & $\begin{array}{l}\text { Fatores mecânicos / Umidade / } \\
\text { Pressão sobre saliência óssea }\end{array}$ & \\
\hline Integridade da pele prejudicada & 27 & 14,7 & $\begin{array}{l}\text { Fatores mecânicos / Umidade / } \\
\text { Alteração no volume de líquidos / } \\
\text { Nutrição inadequada }\end{array}$ & $\begin{array}{l}\text { Alteração na integridade da } \\
\text { pele }\end{array}$ \\
\hline \multicolumn{5}{|l|}{ Domínio 12} \\
\hline \multicolumn{5}{|l|}{ Classe 1} \\
\hline Dor aguda & 177 & 96,2 & $\begin{array}{l}\text { Expressão facial de dor / } \\
\text { Comportamento expressivo / } \\
\text { Autorrelato da dor }\end{array}$ & $\begin{array}{l}\text { Expressão facial de dor / } \\
\text { Evidência de dor usando uma } \\
\text { lista padronizada de } \\
\text { comportamento de dor para } \\
\text { quem não consegue se } \\
\text { comunicar verbalmente }\end{array}$ \\
\hline \multicolumn{5}{|l|}{ Classe 3} \\
\hline Conforto prejudicado & 184 & 100 & $\begin{array}{l}\text { Controle ambiental insuficiente / } \\
\text { Controle situacional insuficiente / } \\
\text { Regime de tratamento / Sintomas } \\
\text { relativos à doença }\end{array}$ & $\begin{array}{l}\text { Incapacidade de relaxar / } \\
\text { Descontentamento com a } \\
\text { situação }\end{array}$ \\
\hline Total & 184 & 100 & & \\
\hline
\end{tabular}

Fonte: Dados coletados pelos pesquisadores, 2020. 


\section{Discussão}

Em relação aos DE encontrados, no domínio Nutrição destacou-se como diagnóstico: a Nutrição desequilibrada menos do que as necessidades corporais $e$ Deglutição prejudicada, tais diagnósticos representam o perfil clínico dos pacientes da amostra do estudo tendo em vista que mais da metade foram vítimas de TCE, apresentando como fatores relacionais a incapacidade de ingerir alimentos e história de alimentação por sonda.

Estudo que buscou avaliar o perfil clínico epidemiológico e os principais diagnósticos de enfermagem aos pacientes internados com acidente vascular cerebral. Evidenciou, também como diagnóstico, a deglutição e nutrição desequilibrada, menos que as necessidades corporais, estando relacionado principalmente à presença de lesão encefálica e do uso de sedação. ${ }^{11}$

O diagnóstico Risco de glicemia instável foi prevalente estando relacionado a ingestão alimentar insuficiente. Estudo realizado em uma UTI do Rio Grande do Sul, encontrou o Risco de glicemia instável com um percentual de $12 \%$ da sua amostra, em consentâneo com a má nutrição do paciente, o DE risco de glicemia instável é justificado devido os déficits nutricionais apresentados pelos pacientes em situações críticas. 12

O índice glicêmico do paciente apresenta maior controle quando gerenciado pela enfermagem, portanto, é importante que o enfermeiro possua conhecimento amplo sobre os algoritmos para ajuste de insulina e ingesta alimentar para proporcionar um cuidado seguro de modo a aumentar a sobrevida do paciente. ${ }^{13}$

Os diagnósticos, Risco de desequilíbrio eletrolítico $e$ Volume de líquidos excessivos tiveram como fatores relacionados a diarreia e mecanismo regulador comprometido. Estudos recentes corroboram com os diagnósticos apresentados neste estudo. Estudo ${ }^{14}$ acerca dos indicadores de qualidade em terapia nutricional evidenciou que a diarreia varia conforme o conceito utilizado para sua definição e pode estar associada a intolerância à dieta. Outros estudos ${ }^{15-18}$ apontaram o risco de desequilíbrio eletrolítico em $32,2 \%, 36,7 \%$ e $23,7 \%$ três estudos relatam o volume de líquido excessivo com $25,5 \%$ 51,6\% e $92,7 \%$ em pacientes adultos.
Os distúrbios hidroeletrolíticos apresentados por pacientes neurológicos têm múltiplas causas, dentre elas, administração de medicações diuréticas, infusão de fluidos, aumento da perda hídrica insensível. Não obstante, é importante considerar todas as variáveis clínicas na avaliação do paciente, com monitorização dos eletrólitos, de modo a preservar a estabilidade do paciente. $\frac{15}{15}$

No Domínio Eliminação e Troca, prevaleceram os diagnósticos Eliminação urinária prejudicada, Constipação, Risco de constipação e Diarreia. Estudo realizado buscando os DE mais prevalentes na unidade identificou a constipação em $33,33 \%$ de sua amostra, o diagnóstico constipação, risco de constipação e diarreia estão diretamente associados ao estado nutricional que os pacientes em situações críticas de saúde apresentam. $\frac{13}{}$ Neste estudo, estiveram relacionados principalmente à ingestão insuficiente de líquidos e mudança dos hábitos alimentares; já o risco de constipação esteve relacionado à motilidade gastrointestinal diminuída.

No domínio Atividade/Repouso foram encontrados como diagnósticos a Mobilidade física prejudicada. Estudo realizado em um hospital público de ensino localizado no interior do Paraná, onde buscou identificar os diagnósticos e intervenções de enfermagem em pacientes neurológicos, obteve o DE de mobilidade física prejudicada em $11,70 \%$ de sua amostra, estando relacionado principalmente a dor e prejuízo neuromuscular, resultado também encontrado no presente estudo. ${ }^{19}$ Neste mesmo domínio, todos os pacientes apresentaram o diagnóstico Risco de perfusão cerebral ineficaz relacionado a lesão cerebral, fato que condiz em outro estudo, este diagnóstico é definido como um risco de redução da circulação do tecido cerebral capaz de comprometer a saúde. ${ }^{20}$ Os diagnósticos déficit de autocuidado para alimentação, déficit de autocuidado para banho, déficit de autocuidado para higiene íntima e déficit de autocuidado para vestir-se esteve presente em $100 \%$ dos pacientes deste estudo, corroborando com estudo desenvolvido em um hospital acadêmico no Nordeste da Itália que encontrou em sua amostra os diagnósticos déficits para autocuidado para banho e higiene em $100 \%$ de sua amostra. ${ }^{21}$ Esses diagnósticos no presente estudo estiveram relacionados a alterações da função cognitiva, desordens perceptivas e prejuízo neuromuscular. 
No domínio Percepção e Cognição, foram prevalentes Confusão aguda em $28 \%$ dos pacientes e Risco de confusão aguda prevalente em $100 \%$ da amostra. A confusão aguda é definida como uma síndrome clínica caracterizada por distúrbios da consciência, função cognitiva ou percepção, sendo frequente em doentes hospitalizados, no presente estudo esteve relacionado a presença de delirium, função metabólica prejudicada, mobilidade prejudicada e alteração da função cognitiva. ${ }^{22}$

No domínio Enfrentamento/Tolerância ao estresse foram identificados os diagnósticos Risco de síndrome do estresse por mudança relacionado a aconseIhamento insuficiente e mudança de ambiente significativa, este diagnóstico mostra a importância dos profissionais de saúde em especial o enfermeiro incluírem em seu plano assistencial todas as demandas apresentadas pelo paciente, possibilitando melhora no processo de enfrentamento. ${ }^{23}$ Outro DE apresentado neste domínio foi Síndrome do trauma por estupro, apresentada por uma das participantes da pesquisa. A síndrome traumática desenvolvida após o estupro, ou sua tentativa, inclui uma fase aguda de desorganização do estilo de vida, onde, consequentemente, se torna um processo prolongado para sua reestruturação. $\underline{\underline{24}}$

No domínio Segurança/Proteção foi evidenciado os seguintes diagnósticos: Risco de infecção, Risco de que$d a$, Risco de úlcera por pressão, Risco de integridade da pele prejudicada, Integridade da pele prejudicada, tais diagnósticos foram apresentados em outras pesquisas. $\frac{2}{2}$ Como o estudo $\underline{25}$ que identificou que pacientes receberam o diagnóstico de integridade da pele prejudicada, na presente pesquisa, este diagnóstico foi apresentado por todos os pacientes investigados, estando relacionado principalmente ao trauma.

Os diagnósticos risco de lesão por pressão e risco de integridade da pele prejudicada estiveram presentes em $100 \%$ da amostra deste estudo. As LP são consideradas um problema de saúde pública que causam uma série de efeitos negativos na recuperação do paciente, provocando o desenvolvimento de outras doenças como infecções, artrite séptica e sepse. ${ }^{26}$ No presente estudo, esteve relacionado a fatores como redução da perfusão tissular, umidade da pele, fatores mecânicos e pressão sobre saliências ósseas.
Diante disto, a prevenção de úlcera por pressão se mostra essencial, pois tem impacto significativo na prevenção de sua incidência e prevalência como também de seus agravos e complicações. $\underline{ }$ ㄱ

O DE risco de infecção esteve relacionado a procedimentos invasivos, considerando o ambiente e o perfil clínico dos pacientes em que o estudo foi desenvolvido, é justificável o fato deste diagnóstico está presente em toda a amostra do estudo, outras pesquisas ${ }^{23}$ desenvolvidas também encontraram alta prevalência deste diagnóstico.

A dor é definida como uma experiência sensorial desagradável associada a lesão tecidual real ou potencial. A identificação do DE dor aguda neste estudo corrobora com outras pesquisas, nas quais foram identificadas o DE dor aguda em $85 \%$ de sua amostra, neste mesmo contexto constatou este mesmo diagnóstico em $33,3 \%$ de sua população estudada. 20,26

Durante a execução desse estudo, foram identificadas algumas limitações como informações incompletas dos pacientes, subnotificação de informações clínicas importantes, dificuldade de acesso aos prontuários, que por não serem informatizados, geraram atrasos na coleta.

\section{Conclusão}

O presente estudo possibilitou a identificação de 25 títulos dos DE da NANDA-I em pacientes neurológicos. Atentando-se aos múltiplos domínios contemplados, devido às alterações cognitivas em consequência da deterioração cerebral.

A identificação dos DE permitiu a caracterização do perfil clínico de pacientes com danos neurológicos, o que proporciona uma avaliação mais direcionada nas reais necessidades dos pacientes com essas afecções. Os diagnósticos de Enfermagem são importantes na condução clínica e refletem sua necessidade, além de contribuir para o fortalecimento da profissão e da Enfermagem como ciência no processo do cuidado, e aproxima o enfermeiro do paciente e das evidências clínicas. 


\section{Contribuições dos autores}

Soares FMM, Mesquita KKB, Teles LESP, Pequeno CLD participaram da concepção, análise e interpretação dos dados. Magalhães DS e Freitas JG participaram da concepção, redação do artigo, revisão crítica do conteúdo intelectual e aprovação da versão final do artigo científico.

\section{Conflito de interesses}

Nenhum conflito financeiro, legal ou político envolvendo terceiros (governo, empresas e fundações privadas, etc.) foi declarado para nenhum aspecto do trabalho submetido (incluindo, mas não se limitando a subvenções e financiamentos, participação em conselho consultivo, desenho de estudo, preparação de manuscrito, análise estatística, etc.).

\section{Referências}

1. Soares FMM, Pequeno CLD, Maia MP, Mariano MR, Abreu RNDC, Sampaio Filho SPC, et al. Perfil clínico de pacientes internados em unidades de neurologia. REAID. 2019;87(25)1-7. https://doi.org/10.31011/reaid-2019-v.87-n.especial-art.162

2. Santos DF, Padula MPC, Waters C. Diagnósticos de enfermagem dos pacientes com Acidente Vascular Cerebral Isquêmico: uma pesquisa bibliográfica. Braz. J. Hea. Rev. 2020;3(1):644-72. https:// doi.org/10.34119/bjhrv3n1-052

3. Lotufo PA, Goulart AC, Passos VMA, Satake FM, Souza MFM, França EB, et al. Doença cerebrovascular no Brasil de 1990 a 2015: Global Burden of Disease 2015. Rev. bras. epidemiol. 2017;20(suppl 1):129-41. https://doi.org/10.1590/19805497201700050011

4. Costa LR, Passos EV, Silvestre OM. Redescobrimento do Brasil Cardiovascular: Como Prevenimos e Tratamos a Doença Cardiovascular em Nosso País. Arq. Bras. Cardiol [Internet]. 2021;116(1):117-8. Disponível em: https://www.scielo.br/j/abc/a/ JDGW77SwZ7Fp4Ld5RDvnrGS/?lang=pt

5. Almeida RGS, Mazzo A, Martins JCA, Jorge BM, Souza JVD, Mendes IAC. Autoconfiança no cuidado ao paciente crítico: pré e pós-intervenção simulada. Rev. Bras. Enferm. 2019;72(6):1618-23. https://doi.org/10.1590/0034-7167-2018-0758

6. Taghavi LT, Saatchi B. Training of NANDA-I Nursing Diagnoses (NDs), Nursing Interventions Classification (NIC) and Nursing Outcomes Classification (NOC), in Psychiatric Wards: A randomized controlled trial. Nurs Open. 2019;6(2):612-9. https:// doi.org/10.1002/nop2.244

7. Horta WA. Processo de enfermagem. São Paulo: EPU; 1979.

8. Alfaro-Lefevre R. Aplicação do processo de enfermagem: Fundamentos para o raciocínio clínico. $7^{\mathrm{a}}$ ed. Porto Alegre: Artmed; 2010.
9. Nanda International Inc. Diagnósticos de enfermagem da NANDA: 2018-2020. Porto Alegre: Artmed; 2020.

10. Oliveira MIV, Bezerra Filho JG, Feitosa RFG, Sousa JEP. Fatores de risco e ideação suicida em pessoas com tentativa de suicídio, Fortaleza, Ceará, Brasil. Rev Baiana Saúde Pública. 2020;12;42(2):262-79. https://doi.org/10.22278/2318-2660.2018. v42.n2.a2598

11. Oliveira J, Damasceno K, Souza L, Lima M. Perfil clínico epidemiológico e os principais rótulos diagnósticos de enfermagem aos pacientes internados com acidente vascular cerebral em um hospital de grande porte na região sul da Amazônia legal. Amazônia Ciência \& Saúde [Internet]. 2016;4(3):311. Disponível em: http://repositorio.facimed.edu.br/xmlui/ handle/123456789/88

12. Ferreira AM, Rocha EN, Lopes CT, Bachion MM, Lopes JL, Barros ALBL. Diagnósticos de enfermagem em terapia intensiva: mapeamento cruzado e Taxonomia da NANDA-I. Rev Bras Enferm. 2016;69(2):285-93. http://dx.doi.org/10.1590/00347167.2016690214i

13. Silva V, Cruz I. Prática de enfermagem baseada em evidência sobre nível de glicemia em UTI - Revisão Sistematizada da Literatura. J. Spec. Nurs. Care [Internet]. 2020;12(1). Disponível em: http://www.jsncare.uff.br/index.php/jsncare/article/ view/3273/819

14. Santos APL, Claudino LM, Pistori MÊS, Mezzomo TR. Indicadores de qualidade em terapia nutricional em uma unidade de terapia intensiva de trauma, Curitiba, PR, Brasil. Nutr. clín. diet. hosp [Internet]. 2018;38(1):149-55. Disponível em: https://revista. nutricion.org/PDF/THAISREGINA.pdf

15. Bittencourt CM, Busanello J, Harter J, Garcia RP. Incidência de volume de líquidos excessivo em pacientes adultos sob cuidados intensivos. Cogitare Enferm. 2021;26.e72689. http://dx.doi. org/10.5380/ce.v26i0.72689

16. Guedes DMB, Rossato LM, Oliveira EA. Diagnóstico de enfermagem mais frequentes em uma unidade de terapia intensiva pediátrica. Rev enferm UFSM. 2015;5(3):476-85. http:// dx.doi.org/10.5902/2179769216471

17. Debone MC, Pedruncci ESN, Candido MCP, Marques S, Kusumota L. Diagnósticos de enfermagem em idosos com doença renal crônica em hemodiálise. Rev. Bras. Enferm. 2017;70(4):8005. https://doi.org/10.1590/0034-7167-2017-0117

18. Hassan A, Mohamed H. Assessment of nurses' knowledge and practice regarding fluids and electrolyte imbalance in critical care units. Port Said Scientific Journal of Nursing. 2021;8(2):1-13. https://dx.doi.org/10.21608/pssjn.2021.180999

19. Rosin J, Matos FGOA, Alves DCl, Carvalho ARS, Lahm JV. Identificação de diagnósticos e intervenções de enfermagem para pacientes neurológicos internados em hospital de ensino. Ciênc cuid saúde. 2016;15(4):607-15. https://doi.org/10.4025/ cienccuidsaude.v15i4.31167 
20. Franco B, Busin L, Chianca TCM, Moraes VM, Pires AUB, Lucena AF. Associações entre discriminadores do Sistema de Triagem de Manchester e diagnósticos de enfermagem. Rev Gaúcha Enferm. 2018;39:e2017-0131. https://doi.org/10.1590/19831447.2018.2017-0131

21. Castellan C, Sluga S, Spina E, Sanson G. Nursing diagnoses, outcomes and interventions as measures of patient complexity and nursing care requirement in Intensive Care Unit. J adv nurs. 2016; 72(6):1273-86. https://doi.org/10.1111/jan.12913

22. Sousa L, Simões C, Araújo I. Prevenção da confusão aguda em doentes adultos internados em cuidados intensivos: Intervenções autónomas do enfermeiro. Revista Portuguesa de Enfermagem de Saúde Mental. 2019;(22):49-57. http://dx.doi.org/10.19131/ rpesm. 0263

23. Santos LF, Oliveira LMCO. Diagnósticos e intervenções de enfermagem em famílias de crianças hospitalizadas. Revista Enfermagem UERJ. 2016;24(4):82-53. https://doi.org/10.12957/ reuerj.2016.8253
24. Guerra AC, Rodrigues CVNR, Carmo DCA, Cruz ICF. Síndrome do trauma de estupro: diagnóstico e prescrição de enfermagem. Boletim NEPAE-NESEN [Internet]. 2017;14(1). Disponível em: http://www.jsncare.uff.br/index.php/bnn/article/view/2946

25. Moura CF, Corgozinho MM, Gomes JRAA. Perfil e Diagnósticos de Enfermagem em Idosos Submetidos ao Tratamento Cirúrgico de Fratura de Fêmur. REVISA [Internet]. 2020;9(3):430-8.

Disponível em: http://revistafacesa.senaaires.com.br/index.php/ revisa/article/view/573

26. Bittencourt C, Carvalho CRC, Busanello J. Diagnósticos de enfermagem prevalentes na internação em unidade de terapia intensiva. SIEPE [Internet]. 2020;11(2). Disponível em: https:// periodicos.unipampa.edu.br/index.php/SIEPE/article/view/101386

27. Macedo ABT, Graciotto A, Mello DB, Hansel LA, Cortelini CSL, Schöninger N. Caracterização das lesões por pressão em adultos portadores de germes multirresistentes. Enfermería Global. 2020;19(3):155-92. https://doi.org/10.6018/eglobal.405051 